\title{
Endophytic Nocardiopsis sp. from Zingiber officinale with both antiphytopathogenic mechanisms and antibiofilm activity against clinical isolates
}

\author{
Rohini Sabu $^{1}$ K. R. Soumya ${ }^{1}$ E. K. Radhakrishnan ${ }^{1}$ (])
}

Received: 27 June 2016/Accepted: 19 April 2017/Published online: 31 May 2017

(C) Springer-Verlag Berlin Heidelberg 2017

\begin{abstract}
Novel and potential antimicrobial compounds are essential to tackle the frequently emerging multidrugresistant pathogens and also to develop environment friendly agricultural practices. In the current study, endophytic actinomycetes from rhizome of Zingiber officinale were explored in terms of its diversity and bioactive properties. Fourteen different organisms were isolated, identified and screened for activity against Pythium myriotylum and human clinical pathogens. Among these, Nocardiopsis sp. ZoA1 was found to have highest inhibition with excellent antibacterial effects compared to standard antibiotics. Remarkable antibiofilm property was also shown by the extract of ZoA1. Its antifungal activity against Pythium and other common phytopathogens was also found to be promising as confirmed by scanning electron microscopic analysis. By PCR-based sequence analysis of $p h z \mathrm{E}$ gene, the organism was confirmed for the genetic basis of phenazine biosynthesis. Further GC-MS analysis of Nocardiopsis sp. revealed the presence of various compounds including Phenol, 2,4-bis (1,1-dimethylethyl) and trans cinnamic acid which can have significant role in the observed result. The current study is the first report on endophytic Nocardiopsis sp. from ginger with promising applications. In vivo treatment of Nocardiopsis sp. on ginger rhizome has revealed its inhibition towards the colonization of $P$. myriotylum which makes the study to
\end{abstract}

Electronic supplementary material The online version of this article (doi:10.1007/s13205-017-0735-4) contains supplementary material, which is available to authorized users.

E. K. Radhakrishnan

radhakrishnanek@mgu.ac.in

1 School of Biosciences, Mahatma Gandhi University, P.D Hills P.O, Kottayam, Kerala 686560, India have promises to manage the severe diseases in ginger like rhizome rot.

Keywords Endophytic actinomycetes - Zingiber officinale · Nocardiopsis sp. · Antibiofilm · Antifungal · GC-MS analysis

\section{Introduction}

The emergence of antibiotic resistant bacteria and other life threatening conditions demand the search for novel and effective chemical scaffolds from unexplored niches. Endophytes are highly promising source for drug discovery. Since the discovery of taxol from the endophytic fungus Taxomyces andreanae, (Stierle et al. 1993) many endophytes from diverse sources have been exploited for various secondary metabolites. Because of its plant-associated nature, these organisms are considered to have the natural mechanisms which can be plant beneficial also. As actinomycetes of endophytic origin can also expected to have strain-specific biosynthetic uniqueness, their bioactivity analysis is very important (Hou et al. 2009). Natural products from actinomycetes have been reported to have an array of properties including antimicrobial (Candicidin, Chloramphenicol, Rifamycin and Streptomycin), antitumor (Actinomycin D, Bleomycin and Doxorubicin), and immunosuppressive effects (Rapamycin and FK 506/Fujimycin) (Arndt et al. 1999). Hence, medicinal plants like Z. officinale (ginger) form excellent source to be explored for actinomycetes with metabolites of both clinical and agricultural importance.

Endophytic actinomycetes have been characterized from a wide range of plants like wheat, rice, potato, tomato, citrus and carrot (Coombs and Franco 2003). Antimycin 
$\mathrm{A}_{18}$, the first naturally occurring antimycin with antifungal activity, was isolated from endophytic Streptomyces sp. recovered from the leaves of Bruguiera gymnorrhiza (Yan et al. 2010). Antibiotics such as alnumycin, munumbicins A to D, and coronamycins, and antitumor drugs such as lupinacidins $\mathrm{A}$ and $\mathrm{B}$, have also been discovered from endophytic actinomycetes (Bieber et al. 1998; Castillo et al. 2002; Igarashi et al. 2007). New bafilomycin derivatives (9-hydroxyl Bafilomycin D and 29-hydroxybafilomycin D) derived from endophytic Streptomyces sp. isolated from Drymaria cordata have been reported to have antitumor activity (Yu et al. 2011). These indicate plants as promising niche for novel and rare endophytic actinomycetes with potential bioactivity.

Zingiber officinale is traditionally known to have broad range of applications including its use to aid digestion and to treat stomach upset, diarrhea, and nausea. From previous reports, constituents of ginger have also shown to have potent antioxidant and anti-inflammatory activities $(\mathrm{Li}$ et al. 2012a), and some of them exhibit cancer preventive activity in experimental carcinogenesis. The presence of [6]-gingerol, [6]-paradol, shogoals and zingerone in ginger are found to be the contributors of its anticancerous property (Zick et al. 2008). Because of this excellent chemical profile, endophytic actinomycetes associated with this plant can also expect to have efficient biosynthetic pathways. Among the various endophytic actinomycetes screened in the current study, a Nocardiopsis sp. was identified to have broad spectrum antifungal and antibacterial activity with promising applications.

\section{Materials and methods}

\section{Isolation of endophytic actinomycetes}

The rhizomes of ginger (Z. officinale) collected locally from the field were used as the source of actinomycetes. The rhizomes were surface sterilized and processed as described previously and were placed onto ten different media selective to actinomycetes supplemented with $50 \mu \mathrm{g} / \mathrm{mL}$ of nalidixic acid and $50 \mu \mathrm{g} / \mathrm{mL}$ of nystatin (Jasim et al. 2014b). This was followed by incubation at $28 \pm 2{ }^{\circ} \mathrm{C}$ for 2 weeks. The media used for the isolation were: arginine glycerol agar, casamino acid yeast extract glucose agar (CYD), Gause's synthetic agar, low nutrient mineral salts (LNMS), mineral salts starch casein agar (MSSC), S-agar, starch casein agar, tap water yeast extract agar (TWYE), casamino acid yeast extract dextrose (YCED) agar, and yeast extract malt extract glucose (YMG) media. Actinomycetes growing out from the plant part were purified on Glycerol-asparagine agar (ISP-5) media and pure colonies were transferred to ISP-5 agar slants for preservation.

\section{Identification of the isolated actinomycetes}

The molecular identification of actinomycetes was carried out by $16 \mathrm{~S}$ rDNA sequence based method. Here, the genomic DNA from purified actinomycetes was isolated using commercially available fungal genomic DNA isolation kit (Chromous Biotech). This was used as template for PCR using 16S rDNA specific primers as per previous report (Coombs and Franco 2003). PCR products were visualized on $1.2 \%(\mathrm{w} / \mathrm{v})$ agarose gel and were used for sequencing. The sequence data thus obtained was subjected to similarity search using BLAST analysis.

\section{Screening of antimicrobial activity of endophytic actinomycetes}

Preliminary antibacterial activity of endophytic actinomycetes was tested against Salmonella enterica typhi, Staphylococcus aureus, Bacillus subtilis and Vibrio cholerae by well diffusion method on Muller Hinton agar (MHA) plates. Wells were made on the Muller Hinton agar plates using a gel puncture and the plates were inoculated by swabbing the bacterial pathogens ( 0.5 McFarland turbidity standards) to create a confluent lawn of bacterial growth. Then $40 \mu \mathrm{L}$ of the methanolic extract from each actinomycete was added into each well. For all the activity analysis, crude methanolic extract prepared from all the purified actinomycetes were used.

Antifungal activity was screened against Pythium myriotylum by well diffusion method. Fungal mycelial discs $(5 \mathrm{~mm})$ were placed at the center of the PDA plate. The wells were made in each plate and crude extract of each endophytic actinomycete strain was added separately $(50 \mu \mathrm{L})$ into each well. The plates were incubated at room temperature for 3-5 days. The zone of inhibition between the edge of the fungal colony and the well was measured. The intensity of inhibition was noted as follows: 1-7 mm (+); 8-14 mm (++); 15-21 (+++); >21 mm (++++). As ZoA1 showed highest inhibition against all the pathogens, it was used for further studies.

\section{Amplification and detection of phenazine gene fragments from ZoA1}

The amplification of $p h z \mathrm{E}$ and $p h z \mathrm{~F}$ genes from ZoA1 was carried out using primers phz Ef (5'-GAAGGCGCCA ACTTCGTYATCAA-3 $\left.3^{\prime}\right) / p h z$ Er $\left(5^{\prime}\right.$-GCCYTCGATGA AGTACTCGGTGTG-3') and Ps_up1 (5'-ATCTTCACC 
CCGGTCAACG-3' ${ }^{\prime}$ ) and Ps_low (5'-CCRTAGGCCGGTG AGAAC-3') (Mavrodi et al. 2010; Schneemann et al. 2011), respectively. PCR parameters for the gene phz $\mathrm{E}$ were initial denaturation at $94{ }^{\circ} \mathrm{C}$ for $2 \mathrm{~min}$, followed by 35 cycles of primer annealing at $54.7^{\circ} \mathrm{C}$ for $60 \mathrm{~s}$, primer extension at $72{ }^{\circ} \mathrm{C}$ for $120 \mathrm{~s}$ and denaturation at $94{ }^{\circ} \mathrm{C}$ for $60 \mathrm{~s}$. A final extension was done at $72{ }^{\circ} \mathrm{C}$ for $7 \mathrm{~min}$. PCR parameters for the gene $p h z \mathrm{~F}$ were: initial denaturation at $94{ }^{\circ} \mathrm{C}$ for $2 \mathrm{~min}$, followed by 35 cycles of primer annealing $57{ }^{\circ} \mathrm{C}$ for $60 \mathrm{~s}$, primer extension at $72{ }^{\circ} \mathrm{C}$ for $120 \mathrm{~s}$ and denaturation at $94{ }^{\circ} \mathrm{C}$ for $60 \mathrm{~s}$. PCR products were visualized by $1.5 \%(\mathrm{w} / \mathrm{v})$ agarose gel, sequenced and sequence data was used for BLAST analysis.

\section{Media optimization for bioactive compound production}

Five different media like ISP1, Rice grain solid, King's B, Tryptic soy broth and Pigment production media were inoculated with ZoA1 and incubated at $150 \mathrm{rpm}$ for 12 days at $28{ }^{\circ} \mathrm{C}$. After incubation, the culture supernatant was extracted with ethyl acetate twice and redissolved in methanol. The extract was further used to evaluate its antimicrobial effects by well diffusion method against test organisms.

\section{Fermentation and extraction}

For large scale culture, ZoA1 was inoculated into $3 \mathrm{~L}$ of King's B broth for 12 days at $28{ }^{\circ} \mathrm{C}$ and $120 \mathrm{rpm}$ in a rotary shaker. After the fermentation process, culture supernatant was extracted twice with ethyl acetate and redissolved in $2 \mathrm{~mL}$ of methanol. Then the methanolic extracts were used for further study.

\section{Antibacterial activity of ZoA1 against clinical CoNS isolates}

For antibacterial activity analysis, wells were made on the Muller Hinton agar plates and the plates were inoculated by swabbing the bacterial pathogens. Then $50 \mu \mathrm{L}$ of the methanolic extracts from ZoA1 and standard antibiotics such as vancomycin $(31.25 \mu \mathrm{g} / \mathrm{mL})$, gentamicin $(11.71 \mu \mathrm{g} /$ $\mathrm{mL})$ and ciprofloxacin $(31.25 \mu \mathrm{g} / \mathrm{mL})$ were added to each well. To study the antibacterial activity, two test organisms were included in the study. These were multidrug-resistant biofilm forming coagulase negative Staphylococci, namely S. capitis (sample id-267) and S. haemolyticus (sample id41 ) isolated from clinical samples. These biofilm formers were confirmed for the presence of biofilm-associated genes icaAB, aap, bhp, atle, fbe and embp by PCR-based analysis (unpublished data).

\section{Analysis of MIC of the crude extract of ZoA1}

The minimum inhibitory concentration of crude extract of ZoA1 against S. capitis 267 and S. haemolyticus 41 was determined by standard microbroth dilution method according to the guidelines of the Clinical Laboratory Standards Institute (CLSI) using 96-well microtiter plates (Thermo Scientific Varioskan Flash multimode reader). The coagulase negative Staphylococci were inoculated into TSB and incubated at $37{ }^{\circ} \mathrm{C}$ for $12 \mathrm{~h}$ and were diluted with Tryptic soy broth to get $0.5 \mathrm{McF}$ arland standard bacterial cell density $\left(10^{8} \mathrm{CFU} / \mathrm{mL}\right)$. Subsequently, the cultures were added to twofold dilutions of the crude extract from 2000 to $3.90625 \mu \mathrm{g} / \mathrm{mL}$. The microtitre plates were incubated at $37{ }^{\circ} \mathrm{C}$ for $24 \mathrm{~h}$. The assays were performed in triplicate to confirm the value of MIC against each tested bacteria (Roshmi et al. 2015).

\section{Fluorescence microscopy analysis of CoNS treated with crude extract of ZoA1}

The bacterial viability assay was determined using fluorescence microscopy with fluorescent dye, acridine orange (AO). The fluorescent dye was prepared as per previous report (Thomas et al. 2015). Briefly, the dye was prepared by mixing $0.05 \mathrm{~mL}$ of stock solution of $1 \%$ Acridine orange (HiMedia, India) and $5 \mathrm{~mL}$ of acetate buffer $0.2 \mathrm{M}$ ( $\mathrm{pH}$ 4.0). Crude extract treated and untreated S. capitis 267 and $S$. haemolyticus 41 were placed on a glass slide and dried at $50{ }^{\circ} \mathrm{C}$, fixed with absolute methanol for $2 \mathrm{~min}$ and air dried. The slides were then stained with acridine orange (AO) staining reagent for $1 \mathrm{~min}$, washed under running tap water and air dried. The samples were then observed under fluorescence microscope (OLYMPUS BX43F Fluorescent Microscope).

\section{Antibiofilm activity of ZoA1 extract}

The efficacy of ZoA1 extract to inhibit the formation of biofilm was confirmed using tissue culture plate method (TCP) as per previous report (Thomas et al. 2015). Biofilm forming multidrug-resistant coagulase negative Staphylococci (41 and 267) were inoculated into TSB medium and incubated at $37^{\circ} \mathrm{C}$ for $24 \mathrm{~h}$. The cultures were serially diluted to $1: 100$ in fresh liquid medium and treated with different concentrations of crude extract (3.90625$2000 \mu \mathrm{g} / \mathrm{mL}$ ) and incubated at $37^{\circ} \mathrm{C}$ for $24 \mathrm{~h}$ using 96-well polystyrene microtiter plates. After incubation, the unbound cells were removed by washing the wells with phosphate buffered saline (PBS) twice. The bound cells were then stained with $0.1 \%$ crystal violet for $20 \mathrm{~min}$. Excess stain was rinsed off by thorough washing with deionised water and plates were kept for air drying. For the

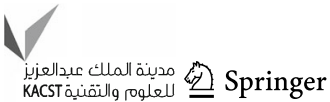


quantification of antibiofilm activity of extract, the adherent bacteria associated with crystal violet were solubilized with $95 \%$ ethanol, and the absorbance were recorded at $590 \mathrm{~nm}$ using microplate reader (Thermo scientific Varioskan Flash Multimode reader). From the OD values, the percentage of biofilm inhibition was calculated by the formula

$$
\begin{gathered}
\% \text { of inhibition }=\text { OD in control }- \text { OD in treatment } / \\
\text { OD in control } \times 100
\end{gathered}
$$

The assay was performed in triplicate. The average value and the standard deviation of the data were calculated and were compared using the Tukey's test. A $p$ value less than 0.05 was considered statistically significant.

\section{Antifungal activity analysis of ZoA1 against phytopathogens}

Broad antifungal activity of ZoA1 was further screened by checking its activity against phytopathogens like Phytophthora infestans, Sclerotium rolfsii, Colletotrichum acutatum, Fusarium oxysporum, Corynespora cassiicola and Rhizoctonia solani in addition to Pythium myriotylum by well diffusion method. Fungal mycelial discs $(5 \mathrm{~mm})$ were placed in the center of the PDA plate. The wells were made in each plate and crude extract of ZoA1 was added $(50 \mu \mathrm{L})$ into each well. The plates were incubated at room temperature for 3-5 days and the zone of inhibition between the edge of the fungal colony and the well was measured.

To further confirm antifungal activity, scanning electron microscopic (SEM) analysis on morphology of phytopathogens in the presence of extract from ZoA1 was done per previous methods (Furlaneto et al. 2012). Hyphal samples of all phytopathogens were excised from the edge of the antifungal assay and were fixed in $2.5 \%$ glutaraldehyde at $4{ }^{\circ} \mathrm{C}$ for $2 \mathrm{~h}$, washed in phosphate buffered saline (PBS) for 4 times (15 min each), dehydrated in a graded ethanol series $(30,50,70,80,90$, and 100\%) (10 min each), air dried and viewed under JEOL 6390 SEM $252 \mathrm{JSM}$ at $20 \mathrm{kV}$ at $3750 \times$ magnification.

\section{Ginger rhizome inoculation effect of ZoA1}

The effect of ZoA1 on ginger rhizome colonization by $P$. myriotylum was studied using previously described methods (Jasim et al. 2014a). Here, surface sterilized rhizome pieces of ginger were soaked in ZoA1 culture extract and the control was treated with sterile water for $1 \mathrm{~h}$. After this, all the rhizome pieces were inoculated with $P$. myriotylum. All the groups were incubated at room temperature and observed periodically for 2 weeks.

\section{GC-MS analysis of extract from Nocardiopsis ZoA1}

For the identification of metabolites from Nocardiopsis ZoA1, gas chromatography-mass spectrometry analysis was carried out with Agilent Technologies-7890 GC System, 5975C inert MSD. $1 \mu \mathrm{L}$ of sample solution was injected into the GC system provided with $30 \mathrm{~m} \times 250 \mu \mathrm{m} \times 0.25 \mu \mathrm{m}$ Agilent 190913-433 column at $325{ }^{\circ} \mathrm{C}$ and helium was used as a carrier gas at a flow rate of $1.1 \mathrm{~mL} / \mathrm{min}$. For GC-MS, the $\mathrm{GC}$ oven was held at $100{ }^{\circ} \mathrm{C}$ and then ramped from 100 to $250{ }^{\circ} \mathrm{C}$ at $5{ }^{\circ} \mathrm{C} / \mathrm{min}$. The chromatogram and mass spectra were recorded and analyzed. The $\mathrm{m} / \mathrm{z}$ peaks representing mass to charge ratio characteristic compounds were compared with those in the mass spectrum of NIST library of the corresponding organic compound.

\section{Results and discussion}

\section{Isolation and identification of endophytic actinomycetes from $Z$. officinale}

Use of various media for the isolation of endophytic actinomycetes from rhizome of $Z$. officinale has resulted in the identification of 14 morphologically distinct isolates named as ZoA1, ZoA2, ZoA3, ZoA4, ZoA5, ZoA6, ZoA7, ZoA8, ZoA9, ZoA10, ZoA 11, ZoA 12, ZoA 13 and ZoA 14. The morphological characteristics of the isolated actinomycetes are depicted in Supplementary data Table 1.

Studies based on 16S rDNA sequencing are effectively used for the rapid identification of actinomycetes (Qin et al. 2009; Zhao et al. 2011). Therefore, in the current study, 16SrDNA sequences of isolated endophytes were identified, subjected to BLAST analysis, and the sequence data was submitted to NCBI (Table 1). The isolates ZoA1 to ZoA11 showed its maximum identity of $99 \%$ to Nocardiopsis sp. (AY336505, Nocardiopsaceae), 100\% to Cellulosimicrobium funkei (JQ659800, Promicromonosporaceae), $100 \%$ to Streptomyces sp. (HQ873936, Streptomycetaceae), 99\% to Brevibacterium sp. (JX490101, Brevibacteriaceae), $99 \%$ to Brevibacterium sp. (AY577816, Brevibacteriaceae), 99\% to Streptomyces sp. (GU238267, Streptomycetaceae), 99\% to Streptomyces sp. (JX244146, Streptomycetaceae) and $100 \%$ to $M i$ cromonospora sp. (JQ782957, Micromonosporaceae), $100 \%$ to Cellulosimicrobium funkei (KC844778, Promicromonosporaceae), 99\% to Kocuria marina (KF306369, Micrococcaceae) and $99 \%$ to Cellulosimicrobium funkei (JQ659800, Promicromonosporaceae), respectively, in BLAST analysis. Even though isolates ZoA3, ZoA6, ZoA7 (Streptomyces sp.) were found to have similarity at the sequence level they differed in their antimicrobial activity. This was the same with respect to ZoA 4 and ZoA5 
Table 1 Summary of BLAST result of endophytic organisms isolated from Z. officinale rhizome

\begin{tabular}{|c|c|c|c|c|}
\hline S. no. & $\begin{array}{l}\text { Name of the isolates with } \\
\text { accession number }\end{array}$ & Closest NCBI match with accession number & $\begin{array}{l}\text { Percentage of } \\
\text { identity }(\%)\end{array}$ & The isolate is identified as \\
\hline 1 & ZoA 1, KC188323 & Nocardiopsis sp. 20030, AY336505 & 99 & Nocardiopsis sp. \\
\hline 2 & ZoA 2, KC188324 & Cellulosimicrobium funkei, JQ659800 & 100 & Cellulosimicrobium funkei 1 \\
\hline 3 & ZoA 3, KC188325 & Streptomyces sp. AML 785, HQ873936 & 100 & Streptomyces sp. 1 \\
\hline 4 & ZoA 4, KC188326 & Brevibacterium iodinum, JX490101 & 99 & Brevibacterium sp. 1 \\
\hline 5 & ZoA 5, KC188327 & Brevibacterium sp. BH, AY577816 & 99 & Brevibacterium sp. 2 \\
\hline 6 & ZoA 6, KC188328 & Streptomyces niger, GU238267 & 99 & Streptomyces sp. 2 \\
\hline 7 & ZoA 7, KC188329 & Streptomyces sp. TRM46629, JX244146 & 99 & Streptomyces sp. 3 \\
\hline 8 & ZoA 8, KC188330 & Micromonospora sp. 10-139, JQ782957 & 100 & Micromonospora sp. \\
\hline 9 & ZoA 9, Not submitted & Cellulosimicrobium funkei strain, KC844778 & 100 & Cellulosimicrobium funkei 2 \\
\hline 10 & ZoA 10, Not submitted & Kocuria marina strain, KF306369 & 99 & Kocuria marina \\
\hline 11 & ZoA 11 , Not submitted & Cellulosimicrobium funkei strain, JQ659800 & 99 & Cellulosimicrobium funkei 3 \\
\hline 12 & ZoA 12, KC188331 & Niastella sp. Gsoil 221, GQ339899 & 97 & Niastella sp. \\
\hline 13 & ZoA 13, KC188332 & Achromobacter xylosoxidans, JQ923444 & 99 & Achromobacter sp. \\
\hline 14 & ZoA 14, KC188333 & Ancylobacter oerskovii NR042655 & 99 & Ancylobacter sp. \\
\hline
\end{tabular}

(Brevibacterium sp.), and ZoA 2, ZoA 9 and ZoA 11(Cellulosimicrobium funkei) which also showed variation in their antimicrobial activity (data not included). However, the isolates ZoA12, ZoA13, ZoA14 were identified to belong to Proteobacteria. ZoA12, ZoA13 and ZoA14 were identified as Niastella sp., Achromobacter xylosoxidans, and Ancylobacter oerskovii, respectively. These results showed the diversity of endophytic actinomycetes inhabiting $Z$. officinale and the use of various media might have favored the isolation of these culturable actinomycetes from the plant.

According to the previous report (Qin et al. 2009), the use of different isolation approaches can increase the diversity of endophytic actinomycetes obtained. All isolates identified in the study were previously reported as endophytic inhabitants from other plants but only few were reported in ginger. Taechowisan et al. (Taechowisan et al. 2005) have reported endophytic actinomycetes from $Z$. officinale with antagonistic activity against phytopathogens like Colletotrichum musae and Fusarium oxysporum. But the actinomycetes reported were strains of Streptomyces sp. only which are known to have endophytic association in many other plants also (Coombs and Franco 2003; Zhao et al. 2011). Identification of diverse actinomycetes from ginger as shown in the current study indicates the actinomycetes diversity present in ginger which can have potential activities. Among the isolates obtained, Micromonospora sp. have previously been identified as endophyte from roots of medicinal plants such as Ainsliaea henryi and Stellera chamaejasme L., (Zhao et al. 2011) roots of Artemisia апnиа (Li et al. 2012b), leaves of Maytenus austroyunnanensis (Qin et al. 2009) and roots of Triticum aestivum L. (Coombs and Franco 2003). Endophytic Brevibacterium halotolerans with growth promoting activities was previously known from the roots of the halophyte, Prosopis strombulifera (Sgroy et al. 2009). Nocardiopsis sp. have also been found as endophyte in stem and root of Maytenus austroyunnanensis, yam bean (Qin et al. 2009; Stamford et al. 2001). Similarly, Cellulosimicrobium sp. was recovered as endophyte from antibiotic cleansed watermelon (Thomas 2011). Streptomyces sp. found as the endophytic inhabitant in Aucuba japonica was reported to produce 7'-demethylnovobiocin and $5{ }^{\prime \prime}$ dimethylnovobiocin (Sasaki et al. 2001). Cyclopentenone derivatives isolated from endophytic Streptomyces sp. from mangrove plant Aegiceras corniculatum were found to have antibacterial, antitumor and apoptotic activities (Lin et al. 2005). Actinomycetes belonging to the same genus identified in the study also have many members isolated as endophytes. The presence of related strains as endophytes in other plants also supports the isolates in this study as endophytes. The novelty of the current study is the identification of diverse actinomycetes from ginger, where only limited genera were previously known as endophytes.

\section{Screening of antimicrobial activity}

Methanolic extract of all the isolates was used to test antagonistic activity against pathogenic bacteria. Except ZoA 6 all the other isolates exhibited antagonistic activity 
against at least one of the test bacterial strains. Isolate ZoA 1 (Nocardiopsis sp.) showed excellent activity against all the pathogenic strains as revealed by the size of the zone of inhibition (Table 2). Streptomyces isolated as endophyte from maize was described to have antimicrobial activity against S. aureus, Micrococcus luteus, B. subtilis and the yeast $C$. albicans (Araújo et al. 2000). Also, endophytic Micromonospora is known to have the ability to produce a new spirotetronate-class polyketide, maklamicin (1) (Igarashi et al. 2011). This compound showed strong to modest antimicrobial activities against M. luteus, Bacillus subtilis, Bacillus cereus, Staphylococcus aureus, and Enterococcus faecalis and was slightly active against $C$. albicans. The bioactivity of endophytic actinomycetes against plant and human pathogens are highly promising. These reports give clue about the presence of promising bioactive compounds in the isolated strains as indicated by their antimicrobial effects.

The crude extract of actinomycetes was also used to check the antifungal activity. On potato dextrose medium, different fungal strains showed different zone of inhibition against crude extract from actinomycetes. The extract obtained from actinomycetes was found to have the ability to inhibit growth of fungi at varying level (Table 2). ZoA1 showed promising antifungal activity against Pythium myriotylum. Similar results have been reported by various authors using extracts from endophytic actinomycetes (Taechowisan et al. 2003; Verma et al. 2009).

\section{Amplification and detection of phenazine gene fragments}

As ZoA1 was found to have broad antibacterial and antifungal activity, it was selected for further study. ZoA1 was PCR positive for $p h z$ E gene fragment and this was further confirmed by BLAST and phylogenetic analysis where it showed maximum similarity to 2-amino-2-deoxyisochorismate synthase in the biosynthetic pathway of phenazine. Valliappan et al. (2014) have previously reported the $p h z \mathrm{E}$ gene amplification from Nocardiopsis sp. associated with marine sponges which were found to produce phenazine compounds. The result of the current study also suggests Nocardiopsis sp. (ZoA1) to have the property to produce phenazine compounds. Pseudomonas aeruginosa PNA 1 isolated from rhizosphere have been reported to produce phenazine-1-carboxylic acid and anthranilate with inhibitory effect on growth of $P$. myriotylum (Tambong and Höfte 2001). Our previous studies have also showed the role of endophytic Pseudomonas aeruginosa from ginger itself to have the ability to produce phenazine-1-carboxylic acid to prevent the growth of $P$. myriotylum (Jasim et al. 2014a). Hence, the result obtained is highly significant as it provides the gateway information regarding the possible phenazine mediated growth inhibition shown by the Nocardiopsis sp. identified in the study. Also, this shows metabolites of same family to be distributed among different endophytic microbial members of ginger to act as part of its innate immune mechanism. However, the role of

Table 2 Antimicrobial activity analysis result of endophytic organisms isolated from Z. officinale rhizome. The activity was checked against human pathogenic bacteria and plant pathogenic fungus, Pythium myriotylum

\begin{tabular}{|c|c|c|c|c|c|}
\hline Isolates & S. aureus & S. typhi & V. cholerae & B. subtilis & Pythium myriotylum \\
\hline ZoA 1 & ++ & ++++ & ++++ & ++++ & ++ \\
\hline ZoA 2 & ++ & +++ & ++ & +++ & ++ \\
\hline ZoA 3 & ++ & - & +++ & - & ++ \\
\hline ZoA 4 & ++ & ++ & +++ & ++ & ++ \\
\hline ZoA 5 & ++ & - & ++ & - & ++ \\
\hline ZoA 6 & - & - & - & - & - \\
\hline ZoA 7 & ++ & ++ & ++ & - & + \\
\hline ZoA 8 & +++ & +++ & +++ & +++ & + \\
\hline ZoA 9 & - & - & - & - & - \\
\hline ZoA 10 & - & - & - & - & - \\
\hline ZoA 11 & - & - & - & - & - \\
\hline ZoB 1 & +++ & +++ & +++ & +++ & + \\
\hline ZoB 2 & +++ & +++ & +++ & +++ & + \\
\hline ZoB 3 & +++ & +++ & +++ & +++ & + \\
\hline Control & - & - & - & - & - \\
\hline
\end{tabular}

Antimicrobial activity was rated as follows: no inhibition (-); 1-7 mm (+); 8-14 mm (++); 15-21 (+++); >21 $\mathrm{mm}(++++)$ 
Table 3 Comparative antibacterial activity analysis of endophytic Nocardiopsis sp. ZoA1 cultured in different media

\begin{tabular}{|c|c|c|c|c|c|}
\hline \multirow[t]{2}{*}{ Pathogens } & \multicolumn{5}{|l|}{ Media } \\
\hline & Pigment production $(\mathrm{mm})$ & ISP 1 (mm) & King B (mm) & TSB $(\mathrm{mm})$ & Rice grain $(\mathrm{mm})$ \\
\hline B. subtilis & 12 & 15 & 15 & - & 11 \\
\hline S. aureus & 11 & 13 & 13 & - & 12 \\
\hline S. typhi & - & 18 & 20 & - & 15 \\
\hline V. cholerae & - & 21 & 24 & 11 & 17 \\
\hline S. capitis 267 & 12 & 11 & 17 & - & 15 \\
\hline S. haemolyticus 41 & 15 & 12 & 16 & 13 & 14 \\
\hline
\end{tabular}

The activity was analyzed against common bacterial pathogens of humans

Table 4 Comparative antibacterial activity analysis of extract from ZoA1 and selected antibiotics against S. capitis 267 and S. haemolyticus 41

\begin{tabular}{lll}
\hline Treatments & \multicolumn{2}{l}{ Inhibition zone against pathogens } \\
\cline { 2 - 3 } & S. capitis 267 $(\mathrm{mm})$ & S. haemolyticus $41(\mathrm{~mm})$ \\
\hline Extract of ZoA 1 & 25 & 28 \\
Ciprofloxacin & 29 & 18 \\
Vancomycin & 17 & 18 \\
Gentamycin & 21 & 24 \\
\hline
\end{tabular}

these mechanisms has to be studied in detail to further explore its biocontrol applications.

\section{Media optimization for bioactive compound production}

Optimization of the media plays a significant role in the expression of genes of the metabolic pathways, and thereby production of maximum antimicrobial compounds (Sengupta et al. 2015). In the current study, crude extract prepared from King's B media was found to exhibit highest inhibition against the selected test organisms (Table 3).

\section{Antibacterial effects of extract from Nocardiopsis sp. against clinical isolates of CoNS}

To further expand the application of antimicrobial activity of Nocardiopsis sp, clinical isolates of coagulase negative staphylococci (CoNS) were also selected as test pathogens. The selection was done by considering its isolation from clinical samples from our previous study and also due to the identification of variety of biofilm-associated genes in them. Initial antibacterial activity analysis of the crude extract of Nocardiopsis sp. ZoA1 against CoNS showed its activity against both the CoNS strains used. Standard antibiotics such as gentamicin, vancomycin and ciprofloxacin were used as positive control. Interestingly, the extract of ZoA1 showed significantly higher antibacterial activity against $S$. capitis $267(25 \mathrm{~mm})$ and $S$. haemolyticus $41(28 \mathrm{~mm})$. At the same time, the zone of inhibition observed for S. capitis 267 against vancomycin, ciprofloxacin and gentamicin were 17, 29 and $21 \mathrm{~mm}$, respectively. Similarly, S. haemolyticus 41 showed 18, 24 and $18 \mathrm{~mm}$ zone of inhibition against ciprofloxacin, gentamicin and vancomycin, respectively. The result clearly indicates the enhanced antibacterial activity of extract from ZoA1. This suggests promising antimicrobial potential of extract from ZoA1 (Table 4). Sadigh-Eteghad et al. (Sadigh-Eteghad et al. 2011) have previously reported the diethyl ether extract of endophytic Streptomyces levis to have antimicrobial effect due to formation of $15 \mathrm{~mm}$ zone of inhibition against test organism and the value was less than the results of the present study. Recent reports on broad spectrum antibacterial activity of Nocardiopsis synnemataformans endophytic to Tulsi leaves $(21 \mathrm{~mm})$ is supportive to the results obtained in the current study (Singh and Padmavathy 2015).

\section{Fluorescence microscopy analysis of CoNS treated with crude extract of $\mathrm{ZoA1}$}

To further confirm the antibacterial activity, viability of CoNS cells was analyzed in the presence of Nocardiopsis sp. extract by fluorescence microscopy. Interestingly, the fluorescence image of S. capitis 267 and S. haemolyticus 41 without extract treatment appeared as green due to the presence of viable cells (Fig. 1). However, extract-treated bacterial cells were observed to be red, due to the possible bactericidal action of extract from ZoA1. Manikandan and Wu (Manikandan and $\mathrm{Wu} 2013$ ) have previously reported the application of differential fluorescence method to analyze the viability of cells and is supportive to the results obtained.

\section{Antibiofilm activity of extract of ZoA1}

For the quantification of biofilm detachment in the presence of extract of ZoA1, the biofilm formation by 
Fig. 1 Effect of Nocardiopsis sp. ZoA1 extract on viability of selected coagulase negative Staphylococci analyzed by fluorescence microscopy. a untreated $S$. capitis 267 ,

b ZoA1 treated S. capitis 267, c untreated $S$. haemolyticus 41 and $\mathbf{d}$ ZoA1 treated $S$.

haemolyticus 41
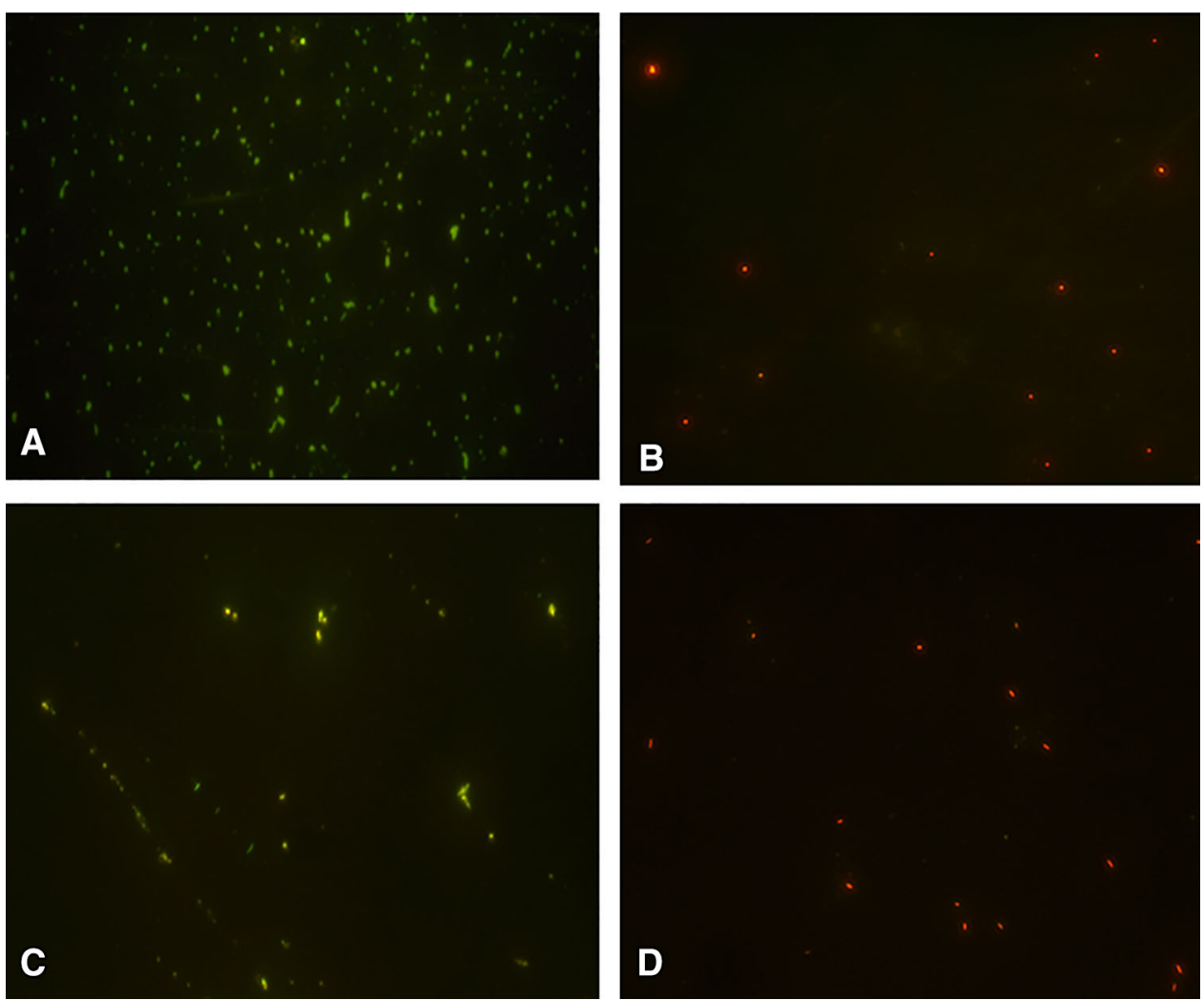

two CoNS strains were evaluated in vitro using 96-well microtitre plates. In this assay, the binding of crystal violet dye to adherent bacterial cells were monitored. This provides direct information regarding the ability of bacteria to form biofilm (Forestier et al. 2013). Both $S$. capitis 267 and $S$. haemolyticus 41 strains were treated with varying concentrations of extract of ZoAl and a dose-dependent biofilm inhibition was observed in all bacteria after $24 \mathrm{~h}$. Crude extract of ZoA1 at $200 \mu \mathrm{g} / \mathrm{mL}$ concentration showed inhibition of more than $90 \%$ of biofilm formation by $S$. capitis 267 , and S. haemolyticus 41. But control experiments of CoNS strains cultured without extract of ZoA1 were found to have formed biofilm and thus extract of Nocardiopsis sp. ZoA1 can be confirmed to have the potential to control biofilm formation by CoNS (Fig. 2).

The dose dependent antibiofilm activity of the extract of ZoA1 as observed again supports the inhibition of synthesis of exopolysaccharide and proteinaceous factors by selected CoNS strains. Bacterial biofilms contain considerable amount of polysaccharides, proteins, nucleic acids and lipids, which are responsible for maintaining the structural integrity of the biofilm and provide an ideal matrix for bacterial cell growth (Sutherland 2001). The broad activity of extract of ZoA1 towards both the polysaccharide and proteinaceous biofilm is noteworthy and it needs more molecular studies to unravel the mechanisms involved. Furthermore, as ZoA1 is an endophyte to $Z$. officinale the chemical basis of the antibiofilm property may expect to have nontoxic effect to humans. Hence, the result of current study indicates the potentiality of the extract of ZoA1 to impede biofilm formation by CoNS, and thus can be used for the treatment of infections caused by multidrug-resistant biofilm forming CoNS.

\section{Antifungal activity analysis of Nocardiopsis sp. ZoA1 against phytopathogens}

To further explore the possible agricultural application of ZoA1, its antifungal activity was analyzed against the common phytopathogens and it was found to have inhibition against all the phytopathogens checked except Sclerotium rolfsii. Scanning electron microscopic examination of treated phytopathogens showed significant morphological changes as confirmatory to the antifungal activity of ZoA1. The most typical features were pronounced collapse and loss of turgor and also deformations of fungal cell wall (Fig. 3). Previously reported Nocardiopsis dassonvillei was shown to have activity against vegetative hyphae of Fusarium oxysporum (El-Tarabily and Sivasithamparam 2006). Similar results have been reported from various 
Fig. 2 Antibiofilm activity of extract of Nocardiopsis sp. ZoA1 analyzed by tissue culture plate method. Increasing concentrations of crude extract of ZoA1 reduced the ability of CoNS to form biofilm and its attachment to the surface of the wells. The results are presented as mean $\pm \mathrm{SD}$. Treated groups showed statistically significant differences from the control group by the Tukey's test $(p<0.05)$

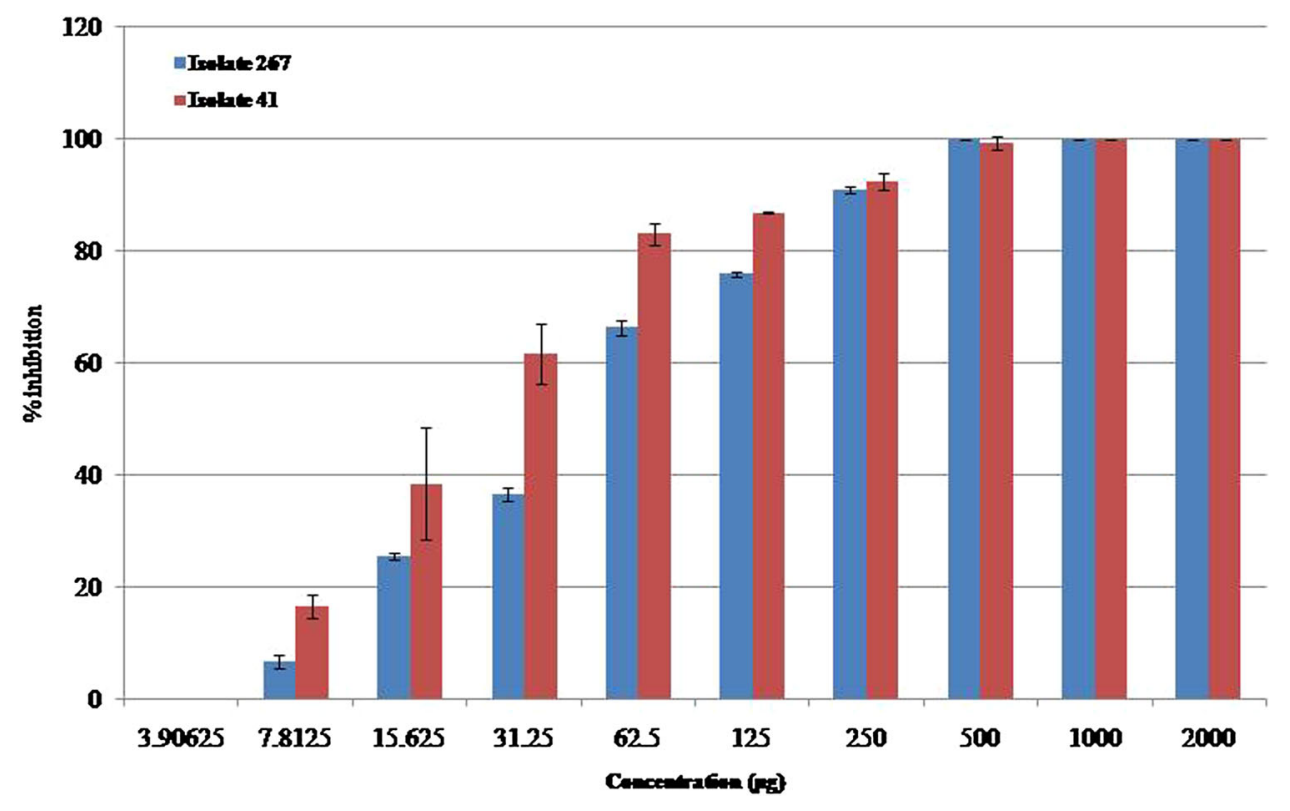

other studies also (Kafur and Khan 2011) where the extract from actinomycetes isolated from Catharanthus roseus was found to be active against $C$. albicans, Botrytis cinerea, Curvularia lunata, Fusarium oxysporum, Fusarium solani and Rhizoctonia solani. Khamna et al. (2008) have reported the extract of actinomycetes to have activity against $R$. stolonifer, A. flavus, F. oxysporum and Alternaria. Streptomyces $\mathrm{sp}$. from $Z$. officinale were reported with inhibitory activity against Colletotrichum musae, and Fusarium oxysporum (Taechowisan et al. 2003). But only limited reports are there on the effect of actinomycetes on Pythium myriotylum which make this result highly significant. This is because $P$. myriotylum is a major causative agent of rhizome rot which cause massive loss of ginger.

\section{Rhizome inoculation effect of ZoA1}

To further study the biocontrol effect of Nocardiopsis sp. ZoA1 against $P$. myriotylum, it was subjected to rhizome inoculation study. The surface sterilized rhizomes of ginger were pre-inoculated with the extract of ZoAl and also with sterile water and this were followed with $P$. myriotylum treatment to study the inhibitory effect of the endophytic constituents on colonization by fungal pathogen. After $48 \mathrm{~h}$ of incubation with $P$. myriotylum only those rhizome pieces which had been pre-treated with water which served as control showed the presence of mycelial growth of $P$. myriotylum while, the rhizome pieces which had been preincubated with ZoA1 extract were found uninfected with $P$. myriotylum even after 2 weeks. This confirmed the inhibitory effect of Nocardiopsis sp. ZoA1 on colonization by $P$. myriotylum on ginger rhizome. It also confirmed endophytic Nocardiopsis from ginger as a promising biocontrol agent to manage rhizome rot in ginger. Further field application of the isolate is required to take steps to explore its potential applications.

\section{GC-MS analysis of Nocardiopsis sp. ZoA1 extract}

Identification of extract of ZoA1 was carried out by GCMS analysis. When compared with NIST database, compounds which were found to have $70 \%$ or more similarity to the hits in database were selected. The retention time, molecular weight and the bioactivity of the compounds corresponding to the five peaks are represented in Fig. 4 and Table 5 and the spectrum of each compound is given in Supplementary data (Fig. 1). Among the five peaks, the presence of phenol, 2,4-bis (1,1-dimethylethyl) and trans cinnamic acid were the two prominent antimicrobial compounds identified in GC-MS analysis. Padmavathi et al. (Padmavathi et al. 2014) have previously reported the phenol, 2,4-bis (1,1-dimethylethyl) from seaweed associated bacterium to have inhibition to quorum sensing mediated biofilm formation in the uropathogen Serratia marcescens. Phenol 2,4-bis (1,1-dimethylethyl) has also been reported as the defense compound of avocado root which prevent the root rot caused by Phytophthora cinnamomi (Rangel-Sanchez et al. 2014). Trans cinnamic acid produced by Photorhabdus luminescens, a nematode symbiont was also identified to be antagonistic to Fusicladium effusum, causal agent of Pecan scab (Bock et al. 2014). All these are supportive to the obtained results and the broad activity of ZoA1 with the expected application in clinical and agricultural fields. 

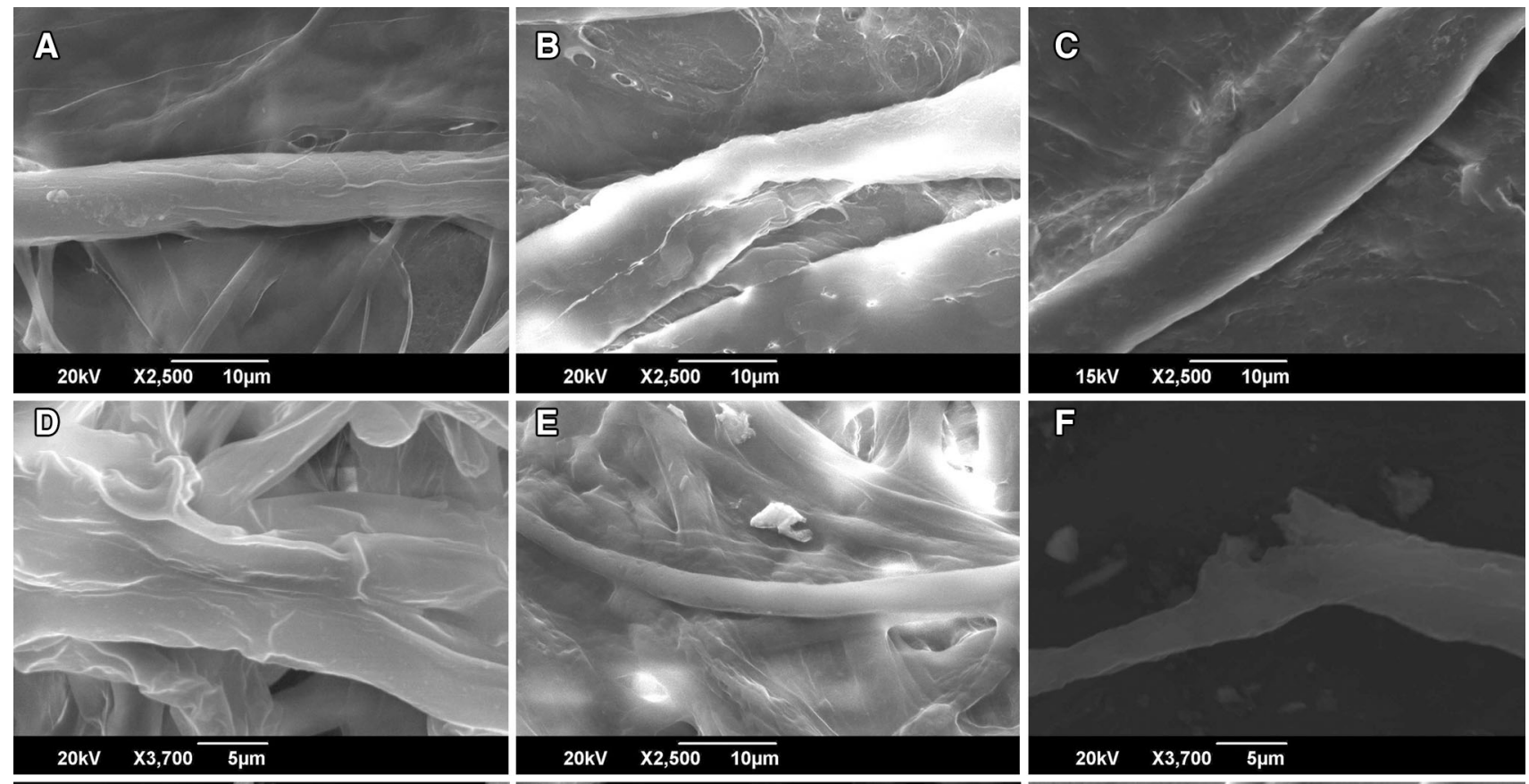

$\boldsymbol{F}$
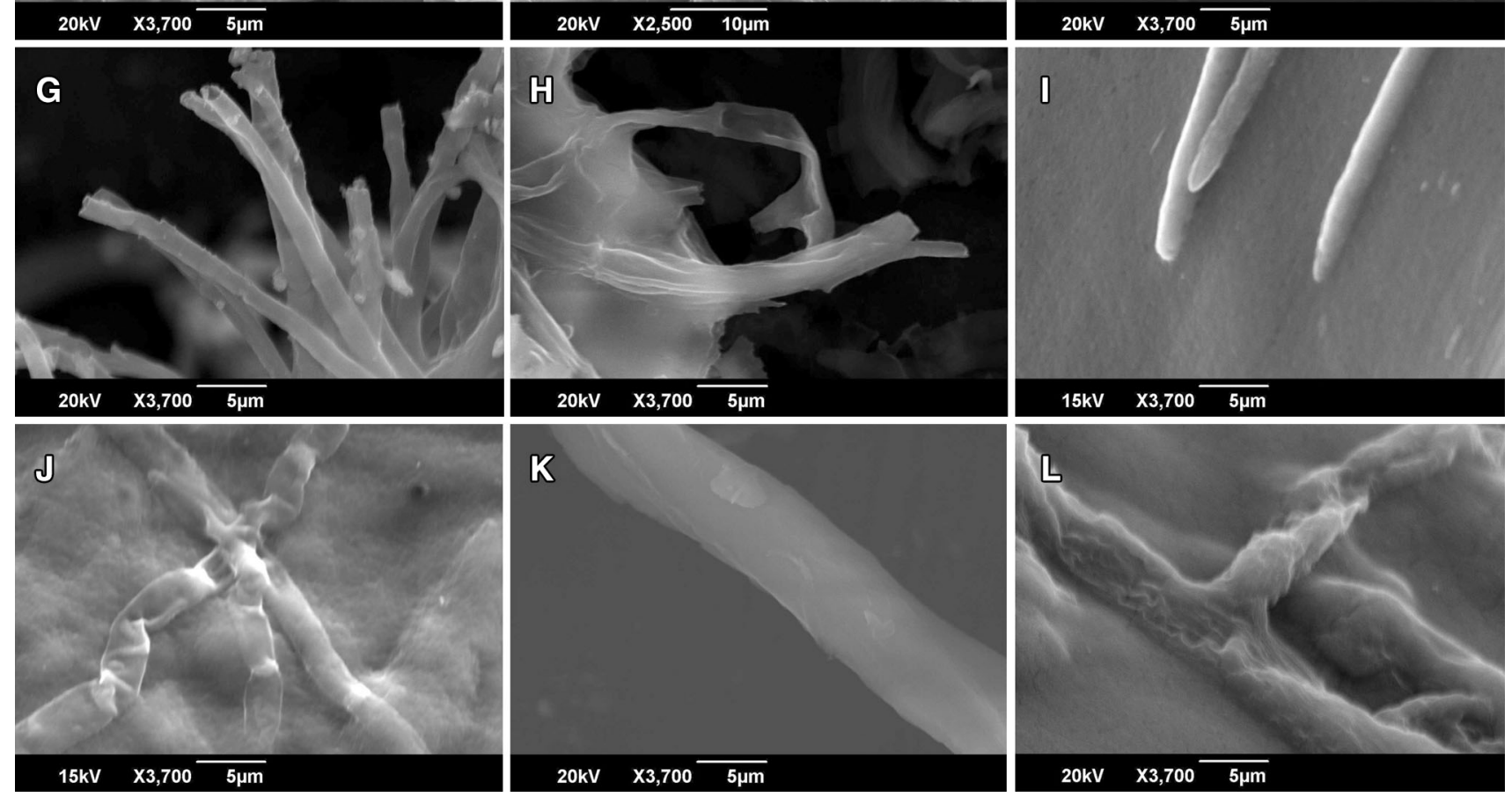

Fig. 3 Effect of extract of Nocardiopsis sp. ZoA1 on the morphological features of phytopathogens analyzed by scanning electron microscopy. a Phytophthora infestans - control, b Phytophthora infestans-treated, c Rhizoctonia solani-control, d Rhizoctonia solani-treated, e Corynespora cassiicola-control, f Corynespora

Nocardiopsis sp. ZoA1 identified in the study was found to have the broad antibacterial, antibiofilm and antiphytopathogenic activity as confirmed by various analysis. Also, from the genome and metabolome analysis, the organism was identified to have the potential to synthesize various chemical scaffolds which might have acted in mechanistically diverse ways to result in multiple and broad activity. cassiicola-treated, g Fusarium oxysporum-control, h Fusarium oxysporum - treated, i Colletotrichum acutatum-control, j Colletotrichum acutatum-treated, k Pythium myriotylum-control, I Pythium myriotylum - treated

\section{Conclusion}

Nature being a sophisticated, versatile and energetic combinatorial chemist makes use of microorganisms as rapid designers of unique and infinite number of exotic and effective structures with broad spectrum activity. The presence of these compounds both in terms of their specific 
Fig. 4 Total ion chromatogram of extract of Nocardiopsis sp. ZoA 1 showing the presence of bioactive compounds at various retention time

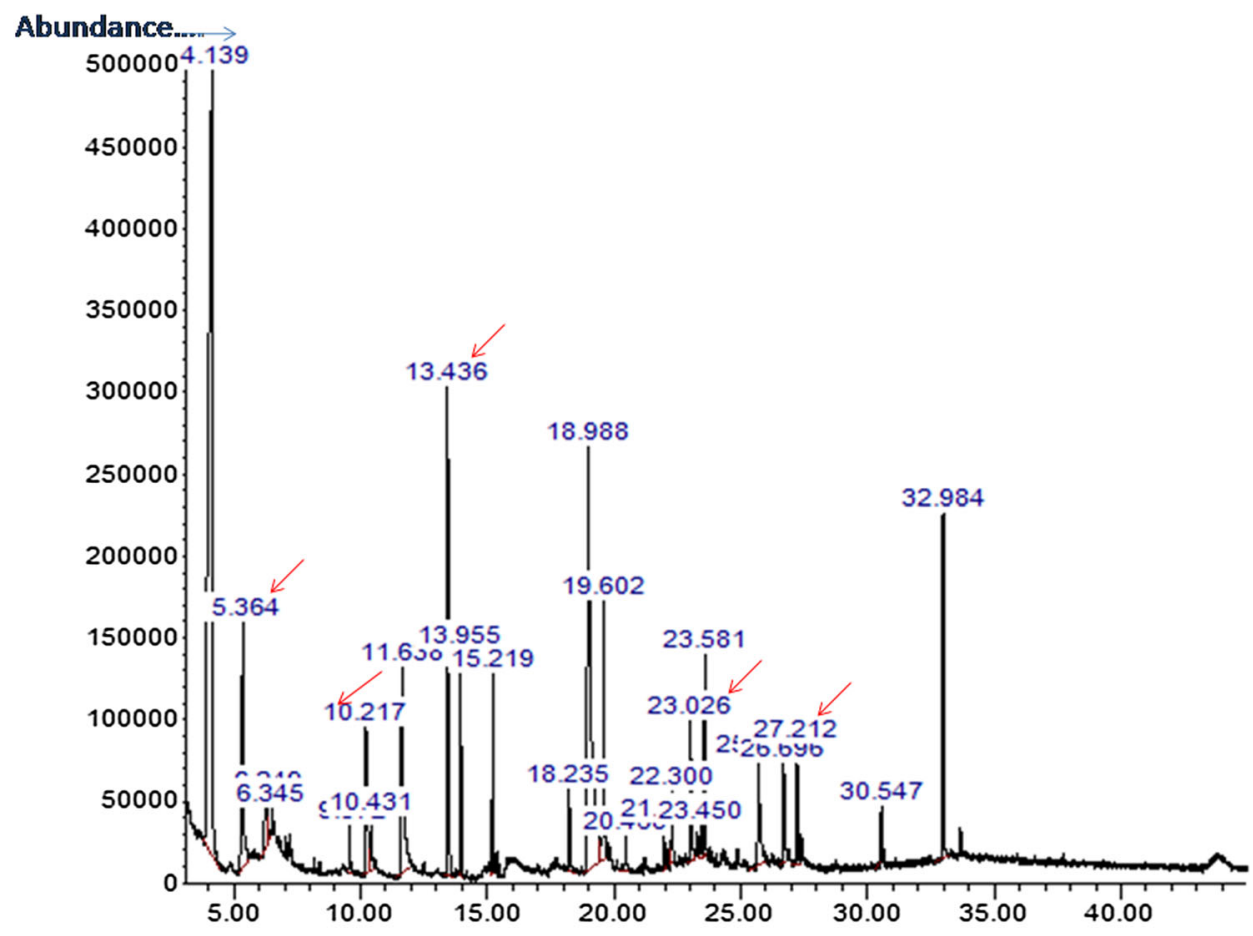

Time->

Table 5 GC-MS based analysis of compounds present in the extract of Nocardiopsis sp. ZoA1

\begin{tabular}{|c|c|c|c|c|c|}
\hline $\begin{array}{l}\text { S. } \\
\text { no. }\end{array}$ & $\begin{array}{l}\text { Retention } \\
\text { time }\end{array}$ & $\begin{array}{l}\% \text { of } \\
\text { similarity }\end{array}$ & $\begin{array}{l}\text { Relative } \\
\text { abundance }(\%)\end{array}$ & Compounds & Reported activity \\
\hline 1 & 5.364 & 94 & 5.07 & Benzoic acid & Antibacterial (Friedman et al. 2003) \\
\hline 2 & 10.216 & 96 & 3.34 & Trans cinnamic acid & Antimicrobial, (Rastogi et al. 1998), antioxidant (Li et al. 2016) \\
\hline 3 & 13.438 & 96 & 7.34 & $\begin{array}{l}\text { Phenol, 2,4-bis }(1,1- \\
\text { dimethylethyl). }\end{array}$ & $\begin{array}{l}\text { Antimicrobial (Rangel-Sánchez et al. 2014) and antioxidant (Lee et al. } \\
\text { 2007) }\end{array}$ \\
\hline 4 & 22.301 & 93 & 0.96 & $n$-Hexadecanoic acid & Antimicrobial (Rahman et al. 2014) \\
\hline 5 & 27.211 & 94 & 1.76 & 1-Heptacosanol & $\begin{array}{l}\text { Nematicidal (Sultana et al. 2010), antimicrobial anticancer and } \\
\text { antioxidant (Murugan and Iyer 2014) }\end{array}$ \\
\hline
\end{tabular}

structure and diversity, make the specific microorganisms to have broad applications. In the study, among the fourteen different organisms studied Nocardiopsis sp. ZoA1 was found to have highest antibacterial, antibiofilm and antiphytopathogenic properties. The organism Nocardiopsis sp. ZoA1 was found to have the presence of $p h z \mathrm{E}$ gene involved in the phenazine biosynthesis. Further GC-MS/ MS analysis of Nocardiopsis sp. revealed the presence of various compounds including phenol, 2,4-bis(1,1-dimethylethyl) and trans cinnamic acid with antimicrobial activity. Hence, from the genomic and metabolite point of view, the organism can be considered to have highly efficient antimicrobial mechanisms. Also, in vivo treatment of
ZoA1 was found to provide impressive protection to ginger rhizome from the colonization of $P$. myriotylum which makes the study highly significant to manage diseases like rhizome rot in ginger.

Acknowledgements The authors express gratitude towards the Kerala State Council for Science, Technology and Environment for the KSCSTE-Fellowship (Council Order No.010, 40/FSHP/2010CSTE) and KSCSTE-SRS project for the funding. The authors also thank School of Chemical sciences and DBT-MSUB project for instrumentation facilities.

\section{Compliance with ethical standards}

Conflict of interest The authors have no conflict of interest. 


\section{References}

Araújo JM, Silva ACD, Azevedo JL (2000) Isolation of endophytic actinomycetes from roots and leaves of maize (Zea mays L.). Brazilian Archives of Biology and Technology 43:0-0

Arndt C, Cruz MC, Cardenas ME, Heitman J (1999) Secretion of FK506/FK520 and rapamycin by Streptomyces inhibits the growth of competing Saccharomyces cerevisiae and Cryptococcus neoformans. Microbiology 145(Pt 8):1989-2000. doi:10. 1099/13500872-145-8-1989

Bieber B, Nuske J, Ritzau M, Grafe U (1998) Alnumycin a new naphthoquinone antibiotic produced by an endophytic Streptomyces sp. J Antibiot (Tokyo) 51:381-382

Bock CH, Shapiro-Ilan DI, Wedge DE, Cantrell CL (2014) Identification of the antifungal compound, trans-cinnamic acid, produced by Photorhabdus luminescens, a potential biopesticide against pecan scab. J Pest Sci 87:155-162. doi:10.1007/s10340013-0519-5

Castillo UF et al (2002) Munumbicins, wide-spectrum antibiotics produced by Streptomyces NRRL 30562, endophytic on Kennedia nigriscans. Microbiology 148:2675-2685. doi:10. 1099/00221287-148-9-2675

Coombs JT, Franco CM (2003) Isolation and identification of actinobacteria from surface-sterilized wheat roots. Appl Environ Microbiol 69:5603-5608

El-Tarabily KA, Sivasithamparam K (2006) Potential of yeasts as biocontrol agents of soil-borne fungal plant pathogens and as plant growth promoters. Mycoscience 47:25-35. doi:10.1007/ s10267-005-0268-2

Forestier C et al (2013) Antibiofilm activity of Actinobacillus pleuropneumoniae serotype 5 capsular polysaccharide. PLoS One 8:e63844. doi:10.1371/journal.pone.0063844

Friedman M, Henika PR, Mandrell RE (2003) Antibacterial activities of phenolic benzaldehydes and benzoic acids against Campylobacter jejuni, Escherichia coli, Listeria monocytogenes, and Salmonella enterica. J Food Prot 66:1811-1821

Furlaneto MC, Andrade CGTJ, Aragão PHA, França EJG, Moralez ATP, Ferreira LCS (2012) Scanning electron microscopy as a tool for the analysis of colony architecture produced by phenotypic switching of a human pathogenic yeast Candida tropicalis. J Phys: Conf Ser 371:012022. doi:10.1088/17426596/371/1/012022

Hou BC et al (2009) Rhizobial resource associated with epidemic legumes in Tibet. Microb Ecol 57:69-81. doi:10.1007/s00248008-9397-4

Igarashi $Y$ et al (2007) Antitumor anthraquinones from an endophytic actinomycete Micromonospora lupini sp. nov. Bioorg Med Chem Lett 17:3702-3705. doi:10.1016/j.bmcl. 2007.04.039

Igarashi Y, Ogura H, Furihata K, Oku N, Indananda C, Thamchaipenet A (2011) Maklamicin, an antibacterial polyketide from an endophytic Micromonospora sp. J Nat Prod 74:670-674. doi:10.1021/np100727h

Jasim B, Anisha C, Rohini S, Kurian JM, Jyothis M, Radhakrishnan EK (2014a) Phenazine carboxylic acid production and rhizome protective effect of endophytic Pseudomonas aeruginosa isolated from Zingiber officinale. World J Microbiol Biotechnol 30:1649-1654. doi:10.1007/s11274-013-1582-z

Jasim B, Joseph AA, John CJ, Mathew J, Radhakrishnan EK (2014b) Isolation and characterization of plant growth promoting endophytic bacteria from the rhizome of Zingiber officinale. 3 Biotech 4:197-204. doi:10.1007/s13205-013-0143-3

Kafur A, Khan AB (2011) Isolation of endophytic actinomycetes from Catharanthes roseus (L.) G. Don leaves and their antimicrobial activity. Iran J Biotechnol 9:302-306
Khamna S, Yokota A, Lumyong S (2008) Actinomycetes isolated from medicinal plant rhizosphere soils: diversity and screening of antifungal compounds, indole-3-acetic acid and siderophore production. World J Microbiol Biotechnol 25:649-655. doi:10. 1007/s11274-008-9933-x

Lee YS, Chang ZQ, Oh BC, Park SC, Shin SR, Kim NW (2007) Antioxidant activity, anti-inflammatory activity, and whitening effects of extracts of Elaeagnus multiflora Thunb. J Med Food 10:126-133. doi:10.1089/jmf.2006.145

Li F, Nitteranon V, Tang X, Liang J, Zhang G, Parkin KL, Hu Q (2012a) In vitro antioxidant and anti-inflammatory activities of 1-dehydro-[6]-gingerdione, 6-shogaol, 6-dehydroshogaol and hexahydrocurcumin. Food Chem 135:332-337. doi:10.1016/j. foodchem.2012.04.145

Li J et al (2012b) Isolation and characterization of culturable endophytic actinobacteria associated with Artemisia annиa L. Antonie Van Leeuwenhoek 101:515-527. doi:10.1007/s10482-011-9661-3

Li W, Zhao X, Sun X, Zu Y, Liu Y, Ge Y (2016) Evaluation of antioxidant ability in vitro and bioavailability of trans-cinnamic acid nanoparticle by liquid antisolvent precipitate. J Nanomater 2016:11. doi:10.1155/2016/9518362

Lin W, Li L, Fu H, Sattler I, Huang X, Grabley S (2005) New cyclopentenone derivatives from an endophytic Streptomyces sp. isolated from the mangrove plant Aegiceras comiculatum. J Antibiot (Tokyo) 58:594-598. doi:10.1038/ja.2005.81

Manikandan M, Wu HF (2013) Rapid differentiation and quantification of live/dead cancer cells using differential photochemical behavior of acridine orange. Photochem Photobiol Sci 12:1921-1926. doi:10.1039/c3pp50165e

Mavrodi DV et al (2010) Diversity and evolution of the phenazine biosynthesis pathway. Appl Environ Microbiol 76:866-879. doi:10.1128/AEM.02009-09

Murugan K, Iyer VV (2014) antioxidant activity and gas chromatographic-mass spectrometric analysis of extracts of the marine algae, Caulerpa peltata and Padina Gymnospora. Indian J Pharm Sci 76:548-552

Padmavathi AR, Abinaya B, Pandian SK (2014) Phenol, 2,4-bis(1,1dimethylethyl) of marine bacterial origin inhibits quorum sensing mediated biofilm formation in the uropathogen Serratia marcescens. Biofouling 30:1111-1122. doi:10.1080/08927014. 2014.972386

Qin S et al (2009) Isolation, diversity, and antimicrobial activity of rare actinobacteria from medicinal plants of tropical rain forests in Xishuangbanna, China. Appl Environ Microbiol 75:6176-6186. doi:10.1128/aem.01034-09

Rahman MM, Ahmad SH, Mohamed MTM, Ab Rahman MZ (2014) Antimicrobial Compounds from Leaf Extracts of Jatropha curcas, Psidium guajava, and Andrographis paniculata. Sci World J 2014:635240. doi:10.1155/2014/635240

Rangel-Sanchez G, Castro-Mercado E, Garcia-Pineda E (2014) Avocado roots treated with salicylic acid produce phenol-2,4bis (1,1-dimethylethyl), a compound with antifungal activity. J Plant Physiol 171:189-198. doi:10.1016/j.jplph.2013.07.004

Rastogi N, Goh KS, Horgen L, Barrow WW (1998) Synergistic activities of antituberculous drugs with cerulenin and transcinnamic acid against Mycobacterium tuberculosis. FEMS Immunol Med Microbiol 21:149-157. doi:10.1111/j.1574695X.1998.tb01161.x

Roshmi T, Soumya KR, Jyothis M, Radhakrishnan EK (2015) Effect of biofabricated gold nanoparticle-based antibiotic conjugates on minimum inhibitory concentration of bacterial isolates of clinical origin. Gold Bull 48:63-71. doi:10.1007/s13404-015-0162-4

Sadigh-Eteghad S, Dehnad A, Shanebandi D, Khalili I, Razmarayii N, Namvaran A (2011) Identification and characterization of a Streptomyces sp. isolate exhibiting activity against multidrug- 
resistant coagulase-negative Staphylococci. Vet Res Commun 35:477-486. doi:10.1007/s11259-011-9491-9

Sasaki T, Igarashi Y, Saito N, Furumai T (2001) TPU-0031-A and B, new antibiotics of the novobiocin group produced by Streptomyces sp. TP-A0556. J Antibiot (Tokyo) 54:441-447

Schneemann I, Wiese J, Kunz AL, Imhoff JF (2011) Genetic approach for the fast discovery of phenazine producing bacteria. Mar Drugs 9:772-789. doi:10.3390/md9050772

Sengupta S, Pramanik A, Ghosh A, Bhattacharyya M (2015) Antimicrobial activities of actinomycetes isolated from unexplored regions of Sundarbans mangrove ecosystem. BMC Microbiol 15:1-16. doi:10.1186/s12866-015-0495-4

Sgroy V, Cassan F, Masciarelli O, Del Papa MF, Lagares A, Luna V (2009) Isolation and characterization of endophytic plant growth-promoting (PGPB) or stress homeostasis-regulating (PSHB) bacteria associated to the halophyte Prosopis strombulifera. Appl Microbiol Biotechnol 85:371-381. doi:10.1007/ s00253-009-2116-3

Singh M, Padmavathy S (2015) Nocardiopsis sp 5 endophytic to Tulsi leaves-isolation and antimicrobial activity. Br Microbiol Res J 5:194-202. doi:10.9734/bmrj/2015/13523

Stamford TLM, Stamford NP, Coelho LCBB, Araújo JM (2001) Production and characterization of a thermostable $\alpha$-amylase from Nocardiopsis sp. endophyte of yam bean. Bioresour Technol 76:137-141. doi:10.1016/s0960-8524(00)00089-4

Stierle A, Strobel G, Stierle D (1993) Taxol and taxane production by Taxomyces andreanae, an endophytic fungus of Pacific yew. Science 260:214-216

Sultana N, Akhter M, Khan RA, Afza N, Tareen RB, Malik A (2010) Nematicidal natural products from the aerial parts of Buddleja crispa. Nat Prod Res 24:783-788. doi:10.1080/ 14786410802496846

Sutherland IW (2001) Biofilm exopolysaccharides: a strong and sticky framework. Microbiology 147:3-9. doi:10.1099/ 00221287-147-1-3

Taechowisan T, Peberdy JF, Lumyong S (2003) Isolation of endophytic actinomycetes from selected plants and their antifungal activity. World J Microbiol Biotechnol 19:381-385. doi:10.1023/a:1023901107182
Taechowisan T, Lu C, Shen Y, Lumyong S (2005) Secondary metabolites from endophytic Streptomyces aureofaciens CMUAc130 and their antifungal activity. Microbiology 151:1691-1695. doi:10.1099/mic.0.27758-0

Tambong JT, Höfte M (2001) Phenazines are involved in biocontrol of Pythium myriotylum on cocoyam by Pseudomonas aeruginosa PNA1. Eur J Plant Pathol 107:511-521. doi:10.1023/a: 1011274321759

Thomas P (2011) Intense association of non-culturable endophytic bacteria with antibiotic-cleansed in vitro watermelon and their activation in degenerating cultures. Plant Cell Rep 30:2313-2325. doi:10.1007/s00299-011-1158-z

Thomas R, Soumya KR, Mathew J, Radhakrishnan EK (2015) Inhibitory effect of silver nanoparticle fabricated urinary catheter on colonization efficiency of coagulase negative Staphylococci. J Photochem Photobiol, B 149:68-77. doi:10.1016/j.jphotobiol. 2015.04.034

Valliappan K, Sun W, Li Z (2014) Marine actinobacteria associated with marine organisms and their potentials in producing pharmaceutical natural products. Appl Microbiol Biotechnol 98:7365-7377. doi:10.1007/s00253-014-5954-6

Verma VC, Gond SK, Kumar A, Mishra A, Kharwar RN, Gange AC (2009) Endophytic actinomycetes from Azadirachta indica A. Juss.: isolation, diversity, and anti-microbial activity. Microb Ecol 57:749-756. doi:10.1007/s00248-008-9450-3

Yan L-L et al (2010) Antimycin A18 produced by an endophytic Streptomyces albidoflavus isolated from a mangrove plant. J Antibiot 63:259-261

$\mathrm{Yu} \mathrm{Z}$ et al (2011) Bafilomycins produced by an endophytic actinomycete Streptomyces sp. YIM56209. J Antibiot (Tokyo) 64:159-162. doi:10.1038/ja.2010.147

Zhao K et al (2011) The diversity and anti-microbial activity of endophytic actinomycetes isolated from medicinal plants in Panxi Plateau, China. Curr Microbiol 62:182-190. doi:10.1007/ s00284-010-9685-3

Zick SM et al (2008) Pharmacokinetics of 6-gingerol, 8-gingerol, 10-gingerol, and 6-shogaol and conjugate metabolites in healthy human subjects. Cancer Epidemiol Biomark Prev 17:1930-1936. doi:10.1158/1055-9965.EPI-07-2934 